\title{
USP7 Gene
}

National Cancer Institute

\section{Source}

National Cancer Institute. USP7 Gene. NCI Thesaurus. Code C118237.

This gene is involved in protein deubiquitination. 